\title{
An Algebraic Approach to Find Some Topological Indices of Derived Graphs of the Benzene Ring
}

\author{
Narahari Narasimha Swamy ${ }^{1, *}$ (D), Gowtham Kalkere Jayanna ${ }^{1}$ (D), \\ Chandan Katigenahalli Gangappa ${ }^{1}$ (D), Badekara Sooryanarayana ${ }^{2}$ (D) \\ 1 University College of Science, Tumkur University, Tumakuru, Karnataka State, India-572103 \\ 2 Dr. Ambedkar Institute of Technology, Bengaluru, Karnataka State, India- 560056 \\ * Correspondence: narahari_nittur@yahoo.com (N.N.S.);
}

Received: 8.07.2021; Revised: 25.08.2021; Accepted: 30.08.2021; Published: 18.10.2021

\begin{abstract}
Topological indices play a vital role in understanding the chemical and structural properties of the chemical compounds and nanostructures. By finding the $M$-polynomial of a graph representing a chemical compound, one can obtain the closed forms of some of the commonly known degree-based topological indices of the compound, such as the Zagreb index, general Randić Index and harmonic index. In this article, we obtain the expression for the $M$-polynomial of the derived graphs of the Benzene ring embedded in the $P$-type surface network in $2 \mathrm{D}$, namely the line graph, the subdivision graph, and the line graph of its subdivision. Furthermore, some of the degree-based topological indices are obtained for these graphs using their $M$-polynomials.
\end{abstract}

Keywords: topological indices; benzene ring; $M$-polynomial; subdivision; line graph; para-line graph. (C) 2021 by the authors. This article is an open-access article distributed under the terms and conditions of the Creative Commons Attribution (CC BY) license (https://creativecommons.org/licenses/by/4.0/).

\section{Introduction}

A chemical graph is the representation of a chemical/molecular structure in terms of a graph, such that each of its atoms is represented by a vertex with an edge representing a bond/multiple bonds between two of its atoms. Such a graph $G=(V, E)$ is simple, undirected, finite, and connected. The order and size of $G$ are, respectively, the number of vertices and edges in it. The length of any shortest path between any two vertices $u$ and $v$ in $G$ is called the distance between them and is denoted $d_{G}(u, v)$.

The subdivision graph $S(G)$ of a graph $G$ is obtained by replacing each edge $e=u v$ in $G$ with a vertex, say $w$, of degree two, and then adding two edges of the form $u w$ and $v w$ to it. The line graph $L(G)$ of $G$ is obtained by replacing each edge of $G$ by a vertex and adding edges to it, in such a way that two vertices in $L(G)$ are adjacent if and only if they share a common vertex in $G$. The para-line graph $L(S(G))$ of $G$ is the line graph of its subdivision graph.

As defined by [1], a Benzene ring is embedded in the $P$-type surface $\left(6.8^{2} P\right)$ and is derived by condensing truncated-icosahedral $C_{60}$ molecules. In particular, twelve atoms are removed from each $C_{60}$ molecule in such a way that the remaining 48 atoms, in eight hexagonal rings, have cubic symmetry. Further, each of these is joined to six identical structures in the six cubic face directions so that four eight-sided rings are formed at each juncture. The $2 \mathrm{D}$ representation of the structure, shortly called the Benzene ring, with $m$ rows and $n$ columns and its subdivision graph is as shown in Figure 1. Further, the line graph and the para-line graph of the structure, taking $m=3$ and $n=5$, are depicted in Figure 2. 

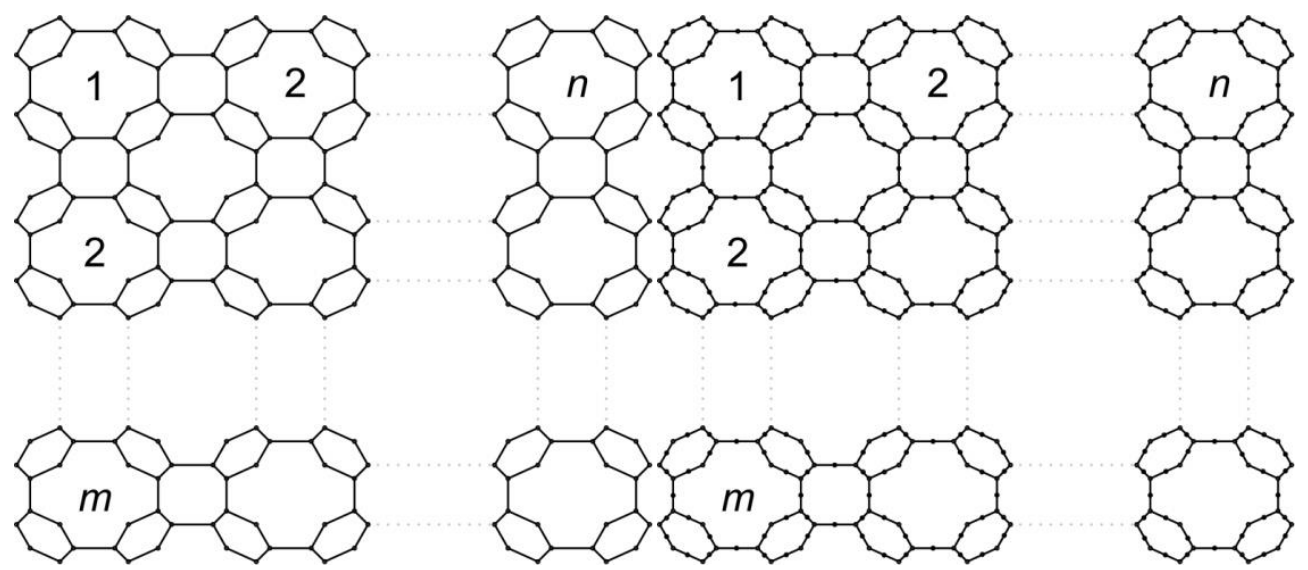

Figure 1. The graphs of the Benzene ring and its subdivision with $m$ rows and $n$ columns.
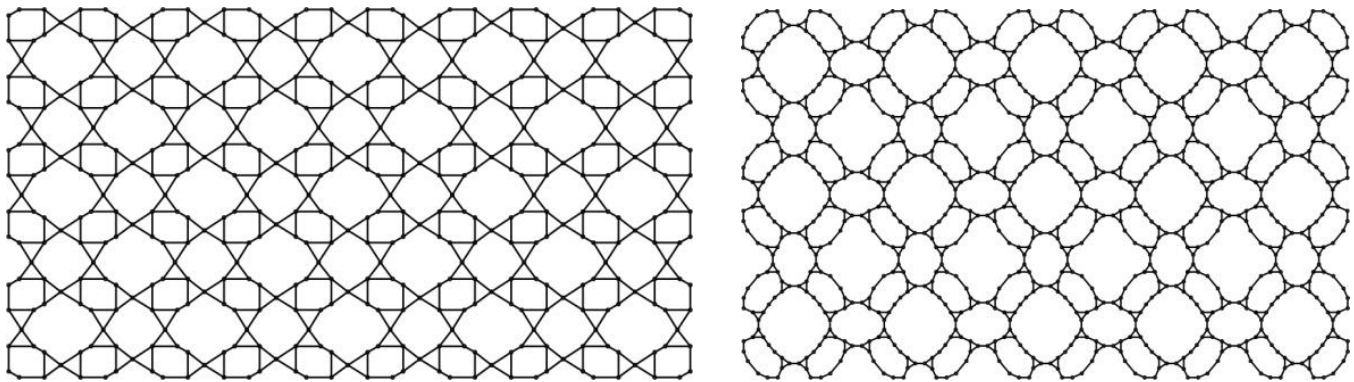

Figure 2. The line and para-line graphs of the Benzene ring with $m=3$ and $n=5$.

A topological index, or a molecular descriptor, of a graph, is a numerical value associated with a graph. It is useful to correlate the structure of the graph with its physical and chemical properties, and it's QSPR/QSAR analysis. For some applications of topological indices, we cite [2-4]. Since the introduction of the first topological index, namely the Wiener index in the year 1947 by Wiener [5], which is based on the topological distance between vertices in a graph, many indices have been formulated, and later generalized, based on the various parameters of the underlying graph such as degree, distance, and spectrum [6-12]. Further, some of these indices have been obtained for particular chemical graphs and nanostructures [13-18]. Wiener index, Hosoya index, Zagreb index, Randić Index and Estrada index are some of the commonly known topological indeces [6, 19, 20].

In the year 1975, M. Randić [6], during his study of the molecular properties of acyclic structures, formulated the Randić index $R_{-1 / 2}(G)$ of a graph $G$ as

$$
R_{-1 / 2}(G)=\sum_{u v \in E(G)} \frac{1}{\sqrt{\operatorname{deg}_{G}(u) \operatorname{deg}_{G}(v)}} .(1)
$$

Later, in the year 1998, Bollobás et al. [21] generalized the Randić index, by replacing $-1 / 2$ by any real number $\alpha$, as the general Randić index $R_{\alpha}(G)$ and the inverse Randić index $R R_{\alpha}(G)$, given by

$$
R_{\alpha}(G)=\sum_{u v \in E(G)}\left(\operatorname{deg}_{G}(u) \operatorname{deg}_{G}(v)\right)^{\alpha}(2)
$$

and

$$
R R_{\alpha}(G)=\sum_{u v \in E(G)} \frac{1}{\left(\operatorname{deg}_{G}(u) \operatorname{deg}_{G}(v)\right)^{\alpha}} .
$$

In the year 1972, Gutman et al. [20] introduced the first and second Zagreb indices, $M_{1}(G)$ and $M_{2}(G)$ of a graph $G$, defined as 


$$
M_{1}(G)=\sum_{u v \in E(G)}\left(\operatorname{deg}_{G}(u)+\operatorname{deg}_{G}(v)\right)(4)
$$

and

$$
M_{2}(G)=\sum_{u v \in E(G)}\left(\operatorname{deg}_{G}(u) \operatorname{deg}_{G}(v)\right) \cdot(5)
$$

Further, the second modified Zagreb index $\quad{ }^{m} M_{2}(G)$ of a graph, $G$ was defined as

$$
{ }^{m} M_{2}(G)=\sum_{u v \in E(G)} \frac{1}{\operatorname{deg}_{G}(u) \operatorname{deg}_{G}(v)} .
$$

The symmetric division index $S D D(G)$ of a graph $G$ was defined by Gupta et al. [22], in the year 2016, as

$$
S D D(G)=\sum_{u v \in E(G)}\left[\frac{\min \left(\operatorname{deg}_{G}(u), \operatorname{deg}_{G}(v)\right)}{\max \left(\operatorname{deg}_{G}(u), \operatorname{deg}_{G}(v)\right)}+\frac{\max \left(\operatorname{deg}_{G}(u), \operatorname{deg}_{G}(v)\right)}{\min \left(\operatorname{deg}_{G}(u), \operatorname{deg}_{G}(v)\right)}\right](7)
$$

Zhong, in the year 2012 [23], introduced a variation of the Randic Index called the harmonic index $H(G)$ of a graph $G$, as

$$
H(G)=\sum_{u v \in E(G)} \frac{2}{\operatorname{deg}_{G}(u)+\operatorname{deg}_{G}(v)} .
$$

As discussed by Vukič ević et al., in the year 2010 [24], the inverse sum index $I(G)$ of a graph $G$ was found to be an important indicator to the total surface area of octane isomers and was defined as

$$
I(G)=\sum_{u v \in E(G)} \frac{\operatorname{deg}_{G}(u) \operatorname{deg}_{G}(v)}{\operatorname{deg}_{G}(u)+\operatorname{deg}_{G}(v)}
$$

Formulated by Huang et al., in the year 2012 [25], the augmented Zagreb index $A(G)$ of a graph $G$, found to be very useful in the study of the heat of formation of octanes and heptanes, is defined as

$$
A(G)=\sum_{u v \in E(G)}\left[\frac{\operatorname{deg}_{G}(u) \operatorname{deg}_{G}(v)}{\operatorname{deg}_{G}(u)+\operatorname{deg}_{G}(v)-2}\right]^{3} .
$$

\section{Materials and Methods}

In literature, the study of some of the topological indices has been done by means of constructing graph-related polynomials. Some of the well-known graph polynomials are the Hosoya polynomial, Tutte polynomial, and the Schlutz polynomial [26-28]. In particular, E. Deutsch et al. [29] introduced the $M$-polynomial as

$$
M(G ; x, y)=\sum_{\delta(G) \leq i \leq j \leq \Delta(G)} m_{i j}(G) x^{i} y^{j}(11)
$$

where $\boldsymbol{\delta}(\boldsymbol{G})$ and $\boldsymbol{\Delta}(\boldsymbol{G})$ are the minimum and maximum degrees of any vertex, respectively, in $\boldsymbol{G}$ and $\boldsymbol{m}_{\boldsymbol{i j}}(\boldsymbol{G})$ is the number of edges $\boldsymbol{e}=\boldsymbol{u} \boldsymbol{v} \in \boldsymbol{E}(\boldsymbol{G})$ such that $\left\{\boldsymbol{d e g}_{\boldsymbol{G}}(\boldsymbol{u}), \boldsymbol{d e g}_{\boldsymbol{G}}(\boldsymbol{v})\right\}=\{\boldsymbol{i}, \boldsymbol{j}\}$. Further, he showed that the closed-form of some of the degree-based topological indices, such as the Zagreb indices, the general Randic Index and harmonic index can be easily obtained using it, as shown in Table 1. Based on this, several authors have worked on computing the polynomials of various chemical graphs and nanostructures, thereby finding their respective indices [30-40].

Table 1. Topological indices in terms of the $M$-polynomial

\begin{tabular}{c|c} 
Topological Index & Expression in terms of $\boldsymbol{M}(\boldsymbol{G} ; \boldsymbol{x}, \boldsymbol{y})$ \\
\hline$M_{1}(G)$ & $\left.\left(D_{x}+D_{y}\right)(M(G ; x, y))\right|_{x=y=1}$ \\
\hline$M_{2}(G)$ & $\left.\left(D_{x} D_{y}\right)(M(G ; x, y))\right|_{x=y=1}$
\end{tabular}




\begin{tabular}{c|c} 
Topological Index & Expression in terms of $\boldsymbol{M}(\boldsymbol{G} ; \boldsymbol{x}, \boldsymbol{y})$ \\
\hline${ }^{m} M_{2}(G)$ & $\left.\left(S_{x} S_{y}\right)(M(G ; x, y))\right|_{x=y=1}$ \\
\hline$R_{\alpha}(G)$ & $\left.\left(D_{x}^{\alpha} D_{y}^{\alpha}\right)(M(G ; x, y))\right|_{x=y=1}$ \\
\hline$R R_{\alpha}(G)$ & $\left.\left(S_{x}^{\alpha} S_{y}^{\alpha}\right)(M(G ; x, y))\right|_{x=y=1}$ \\
\hline$S S D(G)$ & $\left.\left(D_{x} S_{y}+S_{x} D_{y}\right)(M(G ; x, y))\right|_{x=y=1}$ \\
\hline$H$ & $\left.2 S_{x} J(M(G ; x, y))\right|_{x=1}$ \\
\hline$I$ & $\left.S_{x} J D_{x} D_{y}(M(G ; x, y))\right|_{x=1}$ \\
\hline$A$ & $\left.S_{x}^{3} Q_{-2} J D_{x}^{3} D_{y}^{3}(M(G ; x, y))\right|_{x=1}$
\end{tabular}

\begin{tabular}{c|c|c}
$D_{x} f(x, y)=x \partial f(x, y) / \partial x$ & $D_{y} f(x, y)=y \partial f(x, y) / \partial y$ & $S_{x} f(x, y)=\int_{0}^{x}(f(t, y) / t) d t$ \\
\hline$S_{y} f(x, y)=\int_{0}^{y}(f(x, t) / t) d t$ & $J f(x, y)=f(x, x)$ & $Q_{\alpha} f(x, y)=x^{\alpha} f(x, y)$
\end{tabular}

Going in this direction, in this article, we study the $M$-polynomial of some derived graphs of the Benzene ring embedded in the $P$-type surface network in $2 \mathrm{D}$ and its derived graphs, namely the line graph, the subdivision graph, and the line graph of its subdivision. Using these, we obtain these topological indices for each of the corresponding graph structures. For standard terminologies, we refer to [41-43].

\section{Results and Discussion}

In this section, we obtain the closed-form of the $M$-polynomial of subdivision, line graph, and para-line of the Benzene ring embedded in $P$-type surface network, by means of which, we compute their topological indices.

\subsection{M-polynomial of the line graph of the Benzene ring.}

Theorem 3.1 Let $G$ be the line graph of the Benzene ring. Then, the $M$-polynomial of $G$ is given by

$$
M(G[m, n] ; x, y)=4 x^{2} y^{2}+(8 m+8 n-8) x^{2} y^{3}+(8 m n+4) x^{3} y^{3}+(32 m n-
$$
$8 m-8 n) x^{3} y^{4}+(16 m n-8 m-8 n) x^{4} y^{4}$.

Proof. Let $G$ be the line graph of the Benzene ring. Since each of the vertices of $G$ is of degree either two, three or four, the vertex set of $G$ has the following three partitions with respect to a degree:

$V_{\{2\}}(G)=\left\{\left.v \in V(G)\right|_{\operatorname{deg}_{G}(v)=2}\right\}, \quad V_{\{3\}}(G)=\left\{\left.v \in V(G)\right|_{\operatorname{deg}_{G}(v)=3}\right\} \quad$ and $\quad V_{\{4\}}(G)=\{v \in$ $\left.\left.V(G)\right|_{\operatorname{deg}_{G}(v)=4}\right\}$.

Further, the edge set of $G$ has three partitions based on the degree of the end vertices:

$$
\begin{aligned}
& E_{\{2,2\}}(G)=\left\{e=\left.u v \in E(G)\right|_{\operatorname{deg}_{G}(u)=2, \operatorname{deg}_{G}(v)=2}\right\}, \\
& E_{\{2,3\}}(G)=\left\{e=\left.u v \in E(G)\right|_{\operatorname{deg}_{G}(u)=2, \operatorname{deg}_{G}(v)=3}\right\}, \\
& E_{\{3,3\}}(G)=\left\{e=\left.u v \in E(G)\right|_{\operatorname{deg}_{G}(u)=3, \operatorname{deg}_{G}(v)=3}\right\}, \\
& E_{\{3,4\}}(G)=\left\{e=\left.u v \in E(G)\right|_{\operatorname{deg}_{G}(u)=3, \operatorname{deg}_{G}(v)=4}\right\} \text { and } \\
& E_{\{4,4\}}(G)=\left\{e=\left.u v \in E(G)\right|_{\operatorname{deg}_{G}(u)=4, \operatorname{deg}_{G}(v)=4}\right\}, \text { such that }
\end{aligned}
$$

$m_{22}(G)=\left|E_{\{2,2\}}(G)\right|=4, m_{23}(G)=\left|E_{\{2,3\}}(G)\right|=8 m+8 n-8, m_{33}(G)=\left|E_{\{3,3\}}(G)\right|=8 m n+4$, 
$m_{34}(G)=\left|E_{\{3,4\}}(G)\right|=32 m n-8 m-8 \operatorname{nandm}_{44}(G)=\left|E_{\{4,4\}}(G)\right|=16 m n-8 m-8 n$.

Thus, the $M$-polynomial of the given graph is

$$
\begin{aligned}
& M(G ; x, y)=\sum_{i \leq j} m_{i j}(G) x^{i} y^{j} \\
& =m_{22}(G) x^{2} y^{2}+m_{23}(G) x^{2} y^{3}+m_{33}(G) x^{3} y^{3}+m_{34}(G) x^{3} y^{4}+m_{44}(G) x^{4} y^{4} \\
& =4 x^{2} y^{2}+(8 m+8 n-8) x^{2} y^{3}+(8 m n+4) x^{3} y^{3} \\
& +(32 m n-8 m-8 n) x^{3} y^{4}+(16 m n-8 m-8 n) x^{4} y^{4}
\end{aligned}
$$

Theorem 3.2 Let $G$ be the line graph of the Benzene ring. Then,

(1) $M_{1}(G)=400 m n-80 m-80 n$

(2) $M_{2}(G)=712 m n-176 m-176 n+4$

(3) ${ }^{m} M_{2}(G)=\frac{41}{9} m n+\frac{1}{6} m+\frac{1}{6} n+\frac{1}{9}$

(4) $R_{\alpha}(G)=2^{2 \alpha}(4)+3^{\alpha} 2^{\alpha}(8 m+8 n-8)+3^{2 \alpha}(8 m n+4)$

$$
+3^{\alpha} 4^{\alpha}(32 m n-8 m-8 n)+4^{2 \alpha}(16 m n-8 m-8 n)
$$

(5) $R R_{\alpha}(G)=\frac{1}{2^{2^{\alpha}}}(4)+\frac{1}{3^{\alpha} 2^{\alpha}}(8 m+8 n-8)+\frac{1}{3^{2^{\alpha}}}(8 m n+4)$

$$
+\frac{1}{3^{\alpha} 4^{\alpha}}(32 m n-8 m-8 n)+\frac{1}{4^{2 \alpha}}(16 m n-8 m-8 n)
$$

(6) $\operatorname{SSD}(G)=\frac{344}{3} m n-\frac{46}{3} m-\frac{46}{3} n-\frac{4}{3}$

(7) $H(G)=\frac{332}{21} m n-\frac{38}{35} m-\frac{38}{35} n+\frac{2}{15}$

(8) $I(G)=\frac{692}{7} m n-\frac{704}{35} m-\frac{704}{35} n+\frac{2}{5}$

Proof. From Theorem 3.1, we have

$$
\begin{aligned}
M(G ; x, y) & =4 x^{2} y^{2}+(8 m+8 n-8) x^{2} y^{3}+(8 m n+4) x^{3} y^{3} \\
& +(32 m n-8 m-8 n) x^{3} y^{4}+(16 m n-8 m-8 n) x^{4} y^{4} .
\end{aligned}
$$

Then, we have the following:

$$
\begin{gathered}
D_{x} f(x, y)=2(4) x^{2} y^{2}+2(8 m+8 n-8) x^{2} y^{3}+3(8 m n+4) x^{3} y^{3} \\
+3(32 m n-8 m-8 n) x^{3} y^{4}+4(16 m n-8 m-8 n) x^{4} y^{4}, \\
D_{y} f(x, y)=2(4) x^{2} y^{2}+3(8 m+8 n-8) x^{2} y^{3}+3(8 m n+4) x^{3} y^{3} \\
+4(32 m n-8 m-8 n) x^{3} y^{4}+4(16 m n-8 m-8 n) x^{4} y^{4}, \\
D_{y} D_{x} f(x, y)=4(4) x^{2} y^{2}+6(8 m+8 n-8) x^{2} y^{3}+9(8 m n+4) x^{3} y^{3} \\
+12(32 m n-8 m-8 n) x^{3} y^{4}+16(16 m n-8 m-8 n) x^{4} y^{4}, \\
S_{x} S_{y} f(x, y)=\frac{1}{4}(4) x^{2} y^{2}+\frac{1}{6}(8 m+8 n-8) x^{2} y^{3}+\frac{1}{9}(8 m n+4) x^{3} y^{3} \\
+\frac{1}{12}(32 m n-8 m-8 n) x^{3} y^{4}+\frac{1}{16}(16 m n-8 m-8 n) x^{4} y^{4}, \\
D_{x}^{\alpha} D_{y}^{\alpha} f(x, y)=4^{\alpha}(4) x^{2} y^{2}+6^{\alpha}(8 m+8 n-8) x^{2} y^{3}+9^{\alpha}(8 m n+4) x^{3} y^{3} \\
+12^{\alpha}(32 m n-8 m-8 n) x^{3} y^{4}+16^{\alpha}(16 m n-8 m-8 n) x^{4} y^{4}, \\
S_{x}^{\alpha} S_{y}^{\alpha} f(x, y)=\frac{1}{4^{\alpha}}(4) x^{2} y^{2}+\frac{1}{6^{\alpha}}(8 m+8 n-8) x^{2} y^{3}+\frac{1}{9^{\alpha}}(8 m n+4) x^{3} y^{3} \\
+\frac{1}{12^{\alpha}}(32 m n-8 m-8 n) x^{3} y^{4}+\frac{1}{16^{\alpha}}(16 m n-8 m-8 n) x^{4} y^{4},
\end{gathered}
$$




$$
\begin{gathered}
S_{y} D_{x} f(x, y)=4 x^{2} y^{2}+\frac{2}{3}(8 m+8 n-8) x^{2} y^{3}+(8 m n+4) x^{3} y^{3} \\
+\frac{3}{4}(32 m n-8 m-8 n) x^{3} y^{4}+(16 m n-8 m-8 n) x^{4} y^{4}, \\
S_{x} D_{y} f(x, y)=4 x^{2} y^{2}+\frac{3}{2}(8 m+8 n-8) x^{2} y^{3}+(8 m n+4) x^{3} y^{3} \\
+\frac{4}{3}(32 m n-8 m-8 n) x^{3} y^{4}+(16 m n-8 m-8 n) x^{4} y^{4}, \\
2 S_{x} J f(x, y)=2\left[\frac{1}{4}(4) x^{4}+\frac{1}{5}(8 m+8 n-8) x^{5}+\frac{1}{6}(8 m n+4) x^{6}\right. \\
\left.+\frac{1}{7}(32 m n-8 m-8 n) x^{7}+\frac{1}{8}(16 m n-8 m-8 n) x^{8}\right], \\
S_{x} J D_{x} D_{y} f(x, y)=4 x^{4}+\frac{6}{5}(8 m+8 n-8) x^{5}+\frac{9}{6}(8 m n+4) x^{6} \\
+\frac{12}{7}(32 m n-8 m-8 n) x^{7}+\frac{16}{8}(16 m n-8 m-8 n) x^{8}, \\
S_{x}^{3} Q_{-2} J D_{x}^{3} D_{y}^{3} f(x, y)=2^{3}(4) x^{2}+2^{3}(8 m+8 n-8) x^{3}+\frac{3^{6}}{4^{3}}(8 m n+4) x^{4} \\
+\frac{4^{3} 3^{3}}{5^{3}}(32 m n-8 m-8 n) x^{5}+\frac{4^{6}}{6^{3}}(16 m n-8 m-8 n) x^{6}
\end{gathered}
$$

Combining these results, using Table 1, we have the following:

(1) The first Zagreb index

$$
M_{1}(G)=\left.\left(D_{x}+D_{y}\right)(f(x, y))\right|_{x=y=1}=400 m n-80 m-80 n
$$

(2) The second Zagreb index

$$
M_{2}(G)=\left.D_{y} D_{x}(f(x, y))\right|_{x=y=1}=712 m n-176 m-176 n+4
$$

(3) The modified second Zagreb index

$$
{ }^{m} M_{2}(G)=\left.S_{x} S_{y}(f(x, y))\right|_{x=y=1}=\frac{41}{9} m n+\frac{1}{6} m+\frac{1}{6} n+\frac{1}{9}
$$

(4) The generalized Randić index

$$
\begin{aligned}
R_{\alpha}(G)= & \left.D_{x}^{\alpha} D_{y}^{\alpha}(f(x, y))\right|_{x=y=1} \\
=2^{2 \alpha}(4) & +3^{\alpha} 2^{\alpha}(8 m+8 n-8)+3^{2 \alpha}(8 m n+4) \\
& +3^{\alpha} 4^{\alpha}(32 m n-8 m-8 n)+4^{2 \alpha}(16 m n-8 m-8 n)
\end{aligned}
$$

(5) The inverse Randić index

$$
\begin{aligned}
R R_{\alpha}(G) & =\frac{1}{2^{2^{\alpha}}}(4)+\frac{1}{3^{\alpha} 2^{\alpha}}(8 m+8 n-8)+\frac{1}{3^{2^{\alpha}}}(8 m n+4) \\
& +\frac{1}{3^{\alpha} 4^{\alpha}}(32 m n-8 m-8 n)+\frac{1}{4^{2 \alpha}}(16 m n-8 m-8 n)
\end{aligned}
$$

(6) The symmetric division index

$$
\operatorname{SSD}(G)=\frac{344}{3} m n-\frac{46}{3} m-\frac{46}{3} n-\frac{4}{3}
$$

(7) The harmonic index

$$
\begin{gathered}
H(G)=\left.2 S_{x} J f(x, y)\right|_{x=1}=2\left[\frac{1}{4}(4) x^{4}+\frac{1}{5}(8 m+8 n-8) x^{5}+\frac{1}{6}(8 m n+4) x^{6}\right. \\
\left.+\frac{1}{7}(32 m n-8 m-8 n) x^{7}+\frac{1}{8}(16 m n-8 m-8 n) x^{8}\right]_{x=1} \\
=\frac{332}{21} m n-\frac{38}{35} m-\frac{38}{35} n+\frac{2}{15}
\end{gathered}
$$

(8) The inverse sum index

$$
I(G)=\left.S_{x} J D_{x} D_{y} f(x, y)\right|_{x=1}=\left[4 x^{4}+\frac{5}{6}(8 m+8 n-8) x^{5}+\frac{9}{6}(8 m n+4) x^{6}\right.
$$




$$
\begin{aligned}
& \left.+\frac{12}{7}(32 m n-8 m-8 n) x^{7}+\frac{16}{8}(16 m n-8 m-8 n) x^{8}\right]_{x=1} \\
= & \frac{692}{7} m n-\frac{704}{35} m-\frac{704}{35} n+\frac{2}{5}
\end{aligned}
$$

3.2. M-polynomial of the subdivision graph of the Benzene ring.

Theorem 3.3 Let $G_{1}$ be the subdivision graph of the Benzene ring. Then the $M$ polynomial of $G_{1}$ is

$$
M\left(G_{1}[m, n] ; x, y\right)=(16 m n+8 m+8 n) x^{2} y^{2}+(48 m n-12 m-12 n) x^{2} y^{3} .
$$

Proof. Let $G_{1}$ be the subdivision graph of the Benzene ring. Since each of the vertices of $G_{1}$ is of degree either two or three, the vertex set of $G_{1}$ has the following two partitions with respect to a degree:

$V_{\{2\}}\left(G_{1}\right)=\left\{\left.v \in V\left(G_{1}\right)\right|_{\operatorname{deg}_{G_{1}}(v)=2}\right\}$ and $V_{\{3\}}\left(G_{1}\right)=\left\{\left.v \in V\left(G_{1}\right)\right|_{\operatorname{deg}_{G_{1}}(v)=3}\right\}$.

Further, the edge set of $\mathrm{G}_{1}$ has two partitions based on the degree of the end vertices:

$E_{\{2,2\}}\left(G_{1}\right)=\left\{e=\left.u v \in E\left(G_{1}\right)\right|_{\operatorname{deg}_{G_{1}}(u)=2, \operatorname{deg}_{G_{1}}(v)=2}\right\}$ and

$E_{\{2,3\}}\left(G_{1}\right)=\left\{e=\left.u v \in E\left(G_{1}\right)\right|_{\operatorname{deg}_{G_{1}}(u)=2, \operatorname{deg}_{G_{1}}(v)=3}\right\}$, such that,

$m_{22}\left(G_{1}\right)=\left|E_{\{2,2\}}\left(G_{1}\right)\right|=16 m n+8 m+8 n$ and $m_{23}\left(G_{1}\right)=\left|E_{\{2,3\}}\left(G_{1}\right)\right|=48 m n-12 m-12 n$.

Thus, the $M$-polynomial of the given graph is

$$
\begin{gathered}
M\left(G_{1} ; x, y\right)=\sum_{i \leq j} m_{i j}\left(G_{1}\right) x^{i} y^{j}=m_{22}\left(G_{1}\right) x^{2} y^{2}+m_{23}\left(G_{1}\right) x^{2} y^{3} \\
\quad=(16 m n+8 m+8 n) x^{2} y^{2}+(48 m n-12 m-12 n) x^{2} y^{3}
\end{gathered}
$$

Theorem 3.4 Let $G_{1}$ be the subdivision graph of the Benzene ring. Then,

(1) $M_{1}\left(G_{1}\right)=304 m n-28 m-28 n$

(2) $M_{2}\left(G_{1}\right)=352 m n-40 m-40 n$

(3) ${ }^{m} M_{2}\left(G_{1}\right)=12 m n$

(4) $R_{\alpha}\left(G_{1}\right)=2^{2 \alpha}(16 m n+8 m+8 n)+3^{\alpha} 2^{\alpha}(48 m n-12 m-12 n)$

(5) $R R_{\alpha}\left(G_{1}\right)=\frac{1}{2^{2^{\alpha}}}(16 m n+8 m+8 n)+\frac{1}{3^{\alpha} 2^{\alpha}}(48 m n-12 m-12 n)$

(6) $\operatorname{SSD}\left(G_{1}\right)=136 m n-10 m-10 n$

(7) $H\left(G_{1}\right)=\frac{136}{5} m n-4 m-4 n$

(8) $I\left(G_{1}\right)=\frac{368}{5} m n-\frac{32}{5} m-\frac{32}{5} n$

(9) $A\left(G_{1}\right)=512 m n-32 m-32 n$

Proof. From Theorem 3.2, we have

$M\left(G_{1} ; x, y\right)=(16 m n+8 m+8 n) x^{2} y^{2}+(48 m n-12 m-12 n) x^{2} y^{3}$.

Then, we have the following:

$D_{x} f(x, y)=2(16 m n+8 m+8 n) x^{2} y^{2}+2(48 m n-12 m-12 n) x^{2} y^{3}$, 


$$
\begin{aligned}
& D_{y} f(x, y)=2(16 m n+8 m+8 n) x^{2} y^{2}+3(48 m n-12 m-12 n) x^{2} y^{3}, \\
& D_{y} D_{x} f(x, y)=4(16 m n+8 m+8 n) x^{2} y^{2}+6(48 m n-12 m-12 n) x^{2} y^{3}, \\
& S_{x} S_{y} f(x, y)=\frac{1}{4}(16 m n+8 m+8 n) x^{2} y^{2}+\frac{1}{6}(48 m n-12 m-12 n) x^{2} y^{3}, \\
& D_{x}^{\alpha} D_{y}^{\alpha} f(x, y)=4^{\alpha}(16 m n+8 m+8 n) x^{2} y^{2}+6^{\alpha}(48 m n-12 m-12 n) x^{2} y^{3}, \\
& S_{x}^{\alpha} S_{y}^{\alpha} f(x, y)=\frac{1}{4^{\alpha}}(16 m n+8 m+8 n) x^{2} y^{2}+\frac{1}{6^{\alpha}}(48 m n-12 m-12 n) x^{2} y^{3}, \\
& S_{y} D_{x} f(x, y)=(16 m n+8 m+8 n) x^{2} y^{2}+\frac{2}{3}(48 m n-12 m-12 n) x^{2} y^{3}, \\
& S_{x} D_{y} f(x, y)=(16 m n+8 m+8 n) x^{2} y^{2}+\frac{3}{2}(48 m n-12 m-12 n) x^{2} y^{3}, \\
& 2 S_{x} J f(x, y)=2\left[\frac{1}{4}(16 m n+8 m+8 n) x^{4}+\frac{1}{5}(48 m n-12 m-12 n) x^{5}\right], \\
& S_{x} J D_{x} D_{y} f(x, y)=(16 m n+8 m+8 n) x^{4}+\frac{6}{5}(48 m n-12 m-12 n) x^{5}, \\
& S_{x}^{3} Q_{-2} J D_{x}^{3} D_{y}^{3} f(x, y)=8(16 m n+8 m+8 n) x^{2}+8(48 m n-12 m-12 n) x^{3}
\end{aligned}
$$

Using Table 1 and the above expressions, we have

(1) The first Zagreb index

$$
M_{1}\left(G_{1}\right)=\left.\left(D_{x}+D_{y}\right)(f(x, y))\right|_{x=y=1}=304 m n-28 m-28 n
$$

(2) The second Zagreb index

$$
M_{2}\left(G_{1}\right)=\left.D_{y} D_{x}(f(x, y))\right|_{x=y=1}=352 m n-40 m-40 n
$$

(3) The modified second Zagreb index

$$
{ }^{m} M_{2}\left(G_{1}\right)=\left.S_{x} S_{y}(f(x, y))\right|_{x=y=1}=12 m n
$$

(4) The generalized Randić index

$$
R_{\alpha}\left(G_{1}\right)=\left.D_{x}^{\alpha} D_{y}^{\alpha}(f(x, y))\right|_{x=y=1}=2^{2 \alpha}(16 m n+8 m+8 n)+3^{\alpha} 2^{\alpha}(48 m n-12 m-12 n)
$$

(5) The inverse Randić index

$$
R R_{\alpha}\left(G_{1}\right)=\frac{1}{2^{2^{\alpha}}}(16 m n+8 m+8 n)+\frac{1}{3^{\alpha} 2^{\alpha}}(48 m n-12 m-12 n)
$$

(6) The symmetric division index

$$
\operatorname{SSD}\left(G_{1}\right)=136 m n-10 m-10 n
$$

(7) The harmonic index

$$
\begin{gathered}
H\left(G_{1}\right)=\left.2 S_{x} J f(x, y)\right|_{x=1}=2\left[\frac{1}{4}(16 m n+8 m+8 n) x^{4}+\frac{1}{5}(48 m n-12 m-12 n) x^{5}\right]_{x=1} \\
=\frac{136}{5} m n-4 m-4 n
\end{gathered}
$$

(8) The inverse sum index

$$
\begin{gathered}
I\left(G_{1}\right)=\left.S_{x} J D_{x} D_{y} f(x, y)\right|_{x=1}=\left[(16 m n+8 m+8 n) x^{4}+\frac{6}{5}(48 m n-12 m-12 n) x^{5}\right]_{x=1} \\
=\frac{368}{5} m n-\frac{32}{5} m-\frac{32}{5} n
\end{gathered}
$$

(9) The augmented Zagreb index

$$
A\left(G_{1}\right)=\left.S_{x}^{3} Q_{-2} J D_{x}^{3} D_{y}^{3} f(x, y)\right|_{x=1}=[8(16 m n+8 m+8 n)+8(48 m n-12 m-12 n)]_{x=1}
$$




$$
=512 m n-32 m-32 n
$$

3.3. M-polynomial of the para-line graph of the Benzene ring.

Theorem 3.5 Let $G_{2}$ be the para-line graph of the Benzene ring. Then the $M$-polynomial of $G_{2}$ is

$M\left(G_{2}[m, n] ; x, y\right)=(8 m n+8 m+8 n) x^{2} y^{2}+(16 m n) x^{2} y^{3}+(64 m n-18 m-18 n) x^{3} y^{3}$.

Proof. Let $G_{2}$ be the para-line graph of the Benzene ring. Since each of the vertices of $G_{2}$ is of degree either two or three, the vertex set of $G_{2}$ has the following two partitions with respect to a degree:

$V_{\{2\}}\left(G_{2}\right)=\left\{\left.v \in V\left(G_{2}\right)\right|_{\operatorname{deg}_{G_{2}}(v)=2}\right\}$ and $V_{\{3\}}\left(G_{2}\right)=\left\{\left.v \in V(G)\right|_{\operatorname{deg}_{G_{2}}(v)=3}\right\}$.

Further, the edge set of $G_{2}$ has three partitions based on the degree of the end vertices:

$E_{\{2,2\}}\left(G_{2}\right)=\left\{e=\left.u v \in E\left(G_{2}\right)\right|_{\operatorname{deg}_{G_{2}}(u)=2, \operatorname{deg}_{G_{2}}(v)=2}\right\}$,

$E_{\{2,3\}}\left(G_{2}\right)=\left\{e=\left.u v \in E\left(G_{2}\right)\right|_{\operatorname{deg}_{G_{2}}(u)=2, \operatorname{deg}_{G_{2}}(v)=3}\right\}$ and

$E_{\{3,3\}}\left(G_{2}\right)=\left\{e=\left.u v \in E\left(G_{2}\right)\right|_{\operatorname{deg}_{G_{2}}(u)=3, \operatorname{deg}_{G_{2}}(v)=3}\right\}$, such that

$$
\begin{gathered}
m_{22}\left(G_{2}\right)=\left|E_{\{2,2\}}\left(G_{2}\right)\right|=8 m n+8 m+8 n, m_{23}\left(G_{2}\right)=\left|E_{\{2,3\}}\left(G_{2}\right)\right|=16 m n \text { and } \\
m_{33}\left(G_{2}\right)=\left|E_{\{3,3\}}\left(G_{2}\right)\right|=64 m n-18 m-18 n .
\end{gathered}
$$

Thus, the $M$-polynomial of the given graph is

$$
\begin{aligned}
M\left(G_{2} ; x, y\right) & =\sum_{i \leq j} m_{i j}\left(G_{2}\right) x^{i} y^{j}=m_{22}\left(G_{2}\right) x^{2} y^{2}+m_{23}\left(G_{2}\right) x^{2} y^{3}+m_{33}\left(G_{2}\right) x^{3} y^{3} \\
& =(8 m n+8 m+8 n) x^{2} y^{2}+(16 m n) x^{2} y^{3}+(64 m n-18 m-18 n) x^{3} y^{3}
\end{aligned}
$$

Theorem 3.6 Let $G_{2}$ be the para-line graph of the Benzene ring. Then,

(1) $M_{1}\left(G_{2}\right)=496 m n-76 m-76 n$

(2) $M_{2}\left(G_{2}\right)=704 m n-130 m-130 n$

(3) ${ }^{m} M_{2}\left(G_{2}\right)=\frac{106}{9} m n$

(4) $R_{\alpha}\left(G_{2}\right)=2^{2 \alpha}(8 m n+8 m+8 n)+3^{\alpha} 2^{\alpha}(16 m n)+3^{2 \alpha}(64 m n-18 m-18 n)$

(5) $R R_{\alpha}\left(G_{2}\right)=\frac{1}{2^{2^{\alpha}}}(8 m n+8 m+8 n)+\frac{1}{3^{\alpha} 2^{\alpha}}(16 m n)+\frac{1}{3^{2^{\alpha}}}(64 m n-18 m-18 n)$

(6) $S S D\left(G_{2}\right)=\frac{536}{3} m n-20 m-20 n$

(7) $H\left(G_{2}\right)=\frac{476}{15} m n-2 m-2 n$

(8) $I\left(G_{2}\right)=\frac{616}{5} m n-19 m-19 n$

(9) $A\left(G_{2}\right)=921 m n-\frac{4513}{32} m-\frac{4513}{32} n$

Proof. From Theorem 3.3, we have

$M\left(G_{2} ; x, y\right)=(8 m n+8 m+8 n) x^{2} y^{2}+(16 m n) x^{2} y^{3}+(64 m n-18 m-18 n) x^{3} y^{3}$. 
Then, we have the following:

$D_{x} f(x, y)=2(8 m n+8 m+8 n) x^{2} y^{2}+2(16 m n) x^{2} y^{3}+3(64 m n-18 m-18 n) x^{3} y^{3}$,

$D_{y} f(x, y)=2(8 m n+8 m+8 n) x^{2} y^{2}+3(16 m n) x^{2} y^{3}+3(64 m n-18 m-18 n) x^{3} y^{3}$,

$D_{y} D_{x} f(x, y)=4(8 m n+8 m+8 n) x^{2} y^{2}+6(16 m n) x^{2} y^{3}+9(64 m n-18 m-18 n) x^{3} y^{3}$,

$S_{x} S_{y} f(x, y)=\frac{1}{4}(8 m n+8 m+8 n) x^{2} y^{2}+\frac{1}{6}(16 m n) x^{2} y^{3}+\frac{1}{9}(64 m n-18 m-18 n) x^{3} y^{3}$,

$D_{x}^{\alpha} D_{y}^{\alpha} f(x, y)=4^{\alpha}(8 m n+8 m+8 n) x^{2} y^{2}+6^{\alpha}(16 m n) x^{2} y^{3}+9^{\alpha}(64 m n-18 m-18 n) x^{3} y^{3}$,

$S_{x}^{\alpha} S_{y}^{\alpha} f(x, y)=\frac{1}{4^{\alpha}}(8 m n+8 m+8 n) x^{2} y^{2}+\frac{1}{6^{\alpha}}(16 m n) x^{2} y^{3}+\frac{1}{9^{\alpha}}(64 m n-18 m-18 n) x^{3} y^{3}$,

$S_{y} D_{x} f(x, y)=(8 m n+8 m+8 n) x^{2} y^{2}+\frac{2}{3}(16 m n) x^{2} y^{3}+(64 m n-18 m-18 n) x^{3} y^{3}$,

$S_{x} D_{y} f(x, y)=(8 m n+8 m+8 n) x^{2} y^{2}+\frac{3}{2}(16 m n) x^{2} y^{3}+(64 m n-18 m-18 n) x^{3} y^{3}$,

$2 S_{x} J f(x, y)=2\left[\frac{1}{4}(8 m n+8 m+8 n) x^{4}+\frac{1}{5}(16 m n) x^{5}+\frac{1}{6}(64 m n-18 m-18 n) x^{6}\right]$,

$S_{x} J D_{x} D_{y} f(x, y)=(8 m n+8 m+8 n) x^{4}+\frac{6}{5}(16 m n) x^{5}+\frac{9}{6}(64 m n-18 m-18 n) x^{6}$,

and $S_{x}^{3} Q_{-2} J D_{x}^{3} D_{y}^{3} f(x, y)=8(8 m n+8 m+8 n) x^{2}+8(16 m n) x^{3}+\frac{3^{6}}{64}(64 m n-18 m-18 n) x^{4}$

Using Table 1, we have

(1) The first Zagreb index

$$
M_{1}\left(G_{2}\right)=\left.\left(D_{x}+D_{y}\right)(f(x, y))\right|_{x=y=1}=496 m n-76 m-76 n
$$

(2) The second Zagreb index

$$
M_{2}\left(G_{2}\right)=\left.D_{y} D_{x}(f(x, y))\right|_{x=y=1}=704 m n-130 m-130 n
$$

(3) The modified second Zagreb index

$$
{ }^{m} M_{2}\left(G_{2}\right)=\left.S_{x} S_{y}(f(x, y))\right|_{x=y=1}=\frac{106}{9} m n
$$

(4) The generalized Randić index

$$
\begin{aligned}
& R_{\alpha}\left(G_{2}\right)=\left.D_{x}^{\alpha} D_{y}^{\alpha}(f(x, y))\right|_{x=y=1} \\
& =2^{2 \alpha}(8 m n+8 m+8 n)+3^{\alpha} 2^{\alpha}(16 m n)+3^{2 \alpha}(64 m n-18 m-18 n)
\end{aligned}
$$

(5) The inverse Randić index

$$
R R_{\alpha}\left(G_{2}\right)=\frac{1}{2^{2^{\alpha}}}(8 m n+8 m+8 n)+\frac{1}{3^{\alpha} 2^{\alpha}}(16 m n)+\frac{1}{3^{2^{\alpha}}}(64 m n-18 m-18 n)
$$

(6) The symmetric division index

$$
\operatorname{SSD}\left(G_{2}\right)=\frac{536}{3} m n-20 m-20 n
$$

(7) The harmonic index

$$
\begin{aligned}
& H\left(G_{2}\right)=\left.2 S_{x} J f(x, y)\right|_{x=1} \\
& =2\left[\frac{1}{4}(8 m n+8 m+8 n) x^{4}+\frac{1}{5}(16 m n) x^{5}+\frac{1}{6}(64 m n-18 m-18 n) x^{6}\right]_{x=1} \\
& =\frac{476}{15} m n-2 m-2 n
\end{aligned}
$$

(8) The inverse sum index

$$
I\left(G_{2}\right)=\left.S_{x} J D_{x} D_{y} f(x, y)\right|_{x=1}
$$




$$
\begin{aligned}
& =\left[(8 m n+8 m+8 n) x^{4}+\frac{6}{5}(16 m n) x^{5}+\frac{9}{6}(64 m n-18 m-18 n) x^{6}\right]_{x=1} \\
& =\frac{616}{5} m n-19 m-19 n
\end{aligned}
$$

(9) The augmented Zagreb index

$$
\begin{aligned}
& A\left(G_{2}\right)=\left.S_{x}^{3} Q_{-2} J D_{x}^{3} D_{y}^{3} f(x, y)\right|_{x=1} \\
& =\left[2^{3}(8 m n+8 m+8 n) x^{2}+2^{3}(16 m n) x^{3}+\frac{3^{6}}{4^{3}}(64 m n-18 m-18 n) x^{4}\right]_{x=1} \\
& =8(8 m n+8 m+8 n)+8(16 m n)+\frac{3^{6}}{4^{3}}(64 m n-18 m-18 n) \\
& =921 m n-\frac{4513}{32} m-\frac{4513}{32} n
\end{aligned}
$$

\section{Conclusions}

In this paper, we have obtained the closed form of the $M$-polynomial of some derived graphs of the Benzene ring embedded in P-type surface network in 2D. Using these, we have computed some of their degree-based topological indices. As seen in literature, the study of these topological indices, in turn, helps understand many of their physicochemical properties.

\section{Funding}

This research received no external funding.

\section{Acknowledgments}

We are thankful to the management of the University College of Science, Tumkur University, and Dr. Ambedkar Institute of Technology, Bengaluru, for their constant support and encouragement during the preparation of this paper.

\section{Conflicts of Interest}

The authors declare no conflict of interest.

\section{References}

1. O'Keeffe, M.; Adams, G. B.; Sankey, O. F. Predicted New Low Energy Forms of Carbon. Phy. Review Letters 1992, 68, 2325-2330, https://doi.org/10.1103/physrevlett.68.2325.

2. Khadikar, P. V.; Karmarkar, S.; Agrawal, V. K.; Singh, J.; Shrivastava, A.; Lukovits, I.; Diudea, M. V. Szeged index - applications for drug modeling. Letters in Drug Design and Discovery 2005, 2, 606-624, https://doi.org/10.2174/157018005774717334.

3. Natarajan, R.; Kamalakanan,P.; Nirdosh, I. Applications of topological indices to structure-activity relationship modelling and selection of mineral collectors. Indian Journal of Chemistry 2003, 42A, 13301346, http://nopr.niscair.res.in/handle/123456789/20664.

4. Yan, F.; Shang, Q.; Xia, S.; Wang, Q.; Ma, P. Application of Topological Index in Predicting Ionic Liquids Densities by the Quantitative Structure Property Relationship Method. Journal of Chemical \& Engineering data 2015, 60, 734-739, https://doi.org/10.1021/je5008668.

5. Wiener, H. Structural determination of paraffin boiling points. Journal of American Chemical Society 1947, 69, 17-20, https://doi.org/10.1021/ja01193a005.

6. Randić, M. Characterization of molecular branching. Journal of American Chemical Society 1975, 97, 66096615, https://doi.org/10.1021/ja00856a001.

7. Brückler, F. M.; Došlić, T. ; Graovac, A.; Gutman, I. On a class of distance-based molecular structure descriptors. Chemical Physics Letters 2011, 503, 336-338, https://doi.org/10.1016/j.cplett.2011.01.033.

8. Padmakar, V. Khadikar.; Karmarkar, S.; Vijay K.; Agrawal. A Novel PI Index and Its Applications to QSPR/QSAR Studies. J. Chem. Inf. Comput. Sci. 2001, 41,934-949, https://doi:10.1021/ci0003092. 
9. Divyashree, B. K.; Jagadeesh, R.; Siddabasappa Topological Indices of Some Classes of Thorn Complete and Wheel Graphs. Letters in Applied NanoBioScience 2022, 11, https://doi.org/10.33263/LIANBS111.33053321.

10. Fath-Tabar, G.; Furtula, B.; Gutman, I. A new geometric-arithmetic index. J. Math. Chem. 2010, 47, 477, https://doi.org/10.1007/s10910-009-9584-7.

11. Poojary, P.; Raghavendra, A.; Shenoy, B. G.; Farahani, M. R.; Sooryanarayana B. Certain topological indices and polynomials for the Isaac graphs. Journal of Discrete Mathematical Sciences and Cryptography 2021, 24, 511-525, https://doi.org/10.1080/09720529.2021.1896648.

12. Das, K. C.; Trinajstić, N. Comparison between first geometricarithmetic index and atombond connectivity index. Chem. Phys. Lett. 2010, 497, 149-151, https://doi.org/10.1016/j.cplett.2010.07.097

13. Diudea, M. V.; Stefu, M.; Parv B.; John, P. E. Wiener index of armchair polyhex nanotubes. Croat. Chem. Acad. 2004,77, 111-115,https://hrcak.srce.hr/102653

14. Havare, O. C.; Havare, A. K. Computation of the Forgotten Topological Index and Co-Index for Carbon Base Nanomaterial. Polycyclic Aromatic Compounds 2020, https://doi.org/10.1080/10406638.2020.1866621.

15. Mitra K.; D’Silva S. D.; Sooryanarayana B.; Zagreb Alliance Indices. Advances in Mathematics: Scientific Journal 2021, 10, 1273-1284, https://doi.org/10.37418/amsj.10.3.15

16. Stefu, M.; Diudea, M. V. Wiener index of $C_{4} C_{8}$ nanotubes.MATCH Commun. Math Comput. Chem. 2004, 50, 133-144, https://match.pmf.kg.ac.rs/electronic_versions/Match50/match50_133-144.pdf.

17. Baig, A.Q.; Naeem, M.; Gao, W.; Liu, J. B. General fifth M-Zagreb indices and fifth M-Zagreb polynomials of carbon graphite. Eurasian chemical communications 2020, 2,634-640, https://doi.org/10.33945/SAMI/ECC.2020.5.10.

18. Ramane, H. S.; Talwar S. Y.; Gutman, I. Zagreb indices and coindices of total graph, semi-total point graph and semi-total line graph of subdivision graphs.Mathematics Interdisciplinary Research 2020,5, 1-12, https://www.sid.ir/en/journal/ViewPaper.aspx?ID=759797.

19. Hosoya, H. Topological index. A newly proposed quantity characterizing the topological nature of structural isomers of saturated hydrocarbons. Bull. Chem. Soc. Jpn. 1971, 44, 2332-2339, https://doi.org/10.1246/bcsj.44.2332.

20. Gutman, I.; Trinajstić, N. Graph theory and molecular orbitals. Total $\varphi$-electron energy of alternant hydrocarbons. Chem. Phys. Lett. 1972, 17, 535-538, https://doi.org/10.1016/0009-2614(72)85099-1

21. Bollobás, B.; Erdös, P. Graphs of extremal weights. Ars Combin. 1998, 50, 225-233.

22. Gupta, C. K.; Lokesha, V.; Shetty, B. S.; Ranjini, P. S. On the symmetric division deg index of graph. Southeast Asian Bulletin of Mathematics 2016, 40, 59-80.

23. Zhong, L. The harmonic index for graphs. Applied Mathematics Letters 2012, 23, 561-566, https://doi.org/10.1016/j.aml.2011.09.059.

24. Vukič ević D.; Gašperov, M. Bond Additive Modeling 1. Adriatic Indices. Croat. Chem. Acta 2010, 83, 243260, https://hrcak.srce.hr/62202.

25. Huang, Y.; Liu, B.; Gan, L. Augmented Zagreb index of connected graphs. MATCH Commun. Math. Comput. Chem. 2012, 67, 483-494, https://match.pmf.kg.ac.rs/electronic_versions/Match67/n2/match67n2_483494.pdf.

26. Mohamadinezhad-Rashti,H.; Yousefi-Azari, H. Some New Results On the Hosoya Polynomial of Graph Operations. Iranian Journal of Mathematical Chemistry 2010, 1, 37-43, https://dx.doi.org/10.22052/ijmc.2010.5153.

27. Došlić, T. Planar polycyclic graphs and their Tutte polynomials. J. Math. Chem. 2013, 51, 1599-1607, https://doi.org/10.1007/s10910-013-0167-2.

28. Hassani, F.; Iranmanesh A.; Mirzaie, S. Schultz and modified Schultz polynomials of C100 fullerene. MATCH Commun. Math. Comput. Chem. 2013, 69, 87-92, https://match.pmf.kg.ac.rs/electronic_versions/Match69/n1/match69n1_87-92.pdf.

29. Deutsch, E.; Klavžar, S.M-Polynomial and degree-based topological indices. Iranian Journal of Mathematical Chemistry 2015, 6, 93-102, https://dx.doi.org/10.22052/ijmc.2015.10106.

30. Kang, S. M.; Nazeer, W.; Zahid, M. A.; Nizami, A. R.; Aslam, A.; Munir, M. M-polynomials and topological indices of hex-derived networks. Open Phys. 2018, 16, 394-403, https://doi.org/10.1515/phys-2018-0054.

31. Khalaf, A. J. M.; Hussain, S.; Afzal, D.; Afzal, F.; Maqbool, A. M-Polynomial and topological indices of book graph. Journal of Discrete Mathematical Sciences and Cryptography 2020, 23, 1217-1237, https://doi.org/10.1080/09720529.2020.1809115. 
32. Afzal, F.; Hussain, H.; Afzal, D.; Farahani, M. R.; Cancan M.; Ediz, S. On computation of latest topological descriptors of some cactus chains graphs via M-polynomial. Journal of Information and Optimization Sciences 2021, https://doi.org/10.1080/02522667.2021.1896651.

33. Narahari, N.; Sangeetha, T. L.; Sooryanarayana, B. General Fifth M-Zagreb Polynomials of the TUC4C8(R)[p, q] 2D-Lattice and its Derived Graphs.Letters in Applied NanoBioScience 2021,10,1738 1747, https://doi.org/10.33263/LIANBS101.17381747.

34. Raza, Z.;Sukaiti,M.E. M-Polynomial and Degree Based Topological Indices of Some Nanostructures. Symmetry 2020, 12, 831, https://doi.org/10.3390/sym12050831.

35. Afzal, F.; Hussain, S.; Afzal, D.; Hameed, S. M-polynomial and topological indices of zigzag edge coronoid fused by starphene. Open Chemistry 2020, 18, 1362-1369, https://doi.org/10.1515/chem-2020-0161.

36. Cancan, M.; Ediz, S.; Mutee-Ur-Rehman, H.; Afzal, D. M-polynomial and topological indices Poly (EThyleneAmidoAmine) dendrimers. Journal of Information and Optimization Sciences 2020, 1-15, https://doi.org/10.1080/02522667.2020.1745383.

37. Afzal, F.; Hussain, S.; Afzal, D.; Razaq, S. Some new degree based topological indices via M-polynomial. Journal of Information and Optimization Sciences $2020,1-16$, https://doi.org/10.1080/02522667.2020.1744307.

38. Shin, D. Y.; Hussain, S.; Afzal, F.; Park, C.; Afzal, D.; Farahani, M. R. Closed Formulas for Some New Degree Based Topological Descriptors Using M-polynomial and Boron Triangular Nanotube. Frontiers in Chemistry 2021, 8, 1246, https://www.frontiersin.org/article/10.3389/fchem.2020.613873.

39. Das, S.; Rai, S. M-polynomial and related degree-based topological indices of the third type of hex-derived network. Nanosystems : Physics, Chemistry, Mathematics; St. Petersburg 2020,11, 267-274, https://doi.org/10.17586/2220-8054-2020-11-3-267-274.

40. Chu, Y. M.; Imran, M.; Baig, A.Q.; Akhter, S.; Siddiqui, M.K. On M-polynomial-based topological descriptors of chemical crystal structures and their applications. Eur. Phys. J. Plus 2020, 135,874, https://doi.org/10.1140/epjp/s13360-020-00893-9.

41. Buckley, F.; Harary, F.Distance in Graphs, Addison-Wesley, New York, 1990.

42. Gutman, I.; Polansky, O. E. Mathematical Concepts in Organic Chemistry, Springer-Verlag New York, New York, NY, USA, 1986, https://link.springer.com/book/10.1007/978-3-642-70982-1.

43. Harary F.;Graph theory, CRC 1969, https://doi.org/10.1201/9780429493768. 